\title{
News of the Society for Microcirculation
}

\section{Calendar of Events}

October 6-7,1994 Aachen, Germany

November 11-12,1994 Munich, Germany

December 19,1994 Birmingham, UK

January 26-28,1995 Malmö, Sweden

March 25,1995 Basel, Switzerland

March 28-29,1995 Sheffield, UK

June 1995 Florence, Italy

June 28 to July 1,1995 Siena, Italy

September 3-8,1995 London, UK

August 25-31,1996 Munich, Germany

13th Annual Meeting of the German Society for Clinical Microcirculation and Haemorheology

13. Tagung der Gesellschaft für Mikrozirkulation e.V.

Microvascular Research Symposium -'Adaptability of Tissue Microcirculation'

Nordic Microcirculation Society

Swiss Microcirculation Society

British Microcirculation Society

National Congress of the International Society for Microcirculation

9th European Conference on Clinical Haemorheology

Union Internationale de Phlébologie: 12th World Congress

6th World Congress for Microcirculation

Details: Conference Secretariat, Dr. S. Wolf, Augenklinik der Medizinischen Fakultät der

RTWH Aachen, Pauwelstrasse 30, D-52057 Aachen (Germany), Tel. +49 241 8088/351, Fax +49241 i

Details: Prof. M. Menger, Institut für Klinisch-Experimentelle Chirurgie,

Universität des Saarlandes, Oscar-Orth-Strasse,

D-66421 Homburg/Saar (Germany), Tel.+6841 166550, Fax+6841 166553

Details: Dr. S. Egginton, Department of Physiology, University of Birmingham, Medical School, Edgbaston, Birmingham B15 2TT (UK), Tel. +44 (0)121 414 69002, Fax +44 (0)121 4146924

Details: Prof. Bo Risberg, Department of Surgery, Malmö General Hospital, S-214 01 Malmö

(Sweden), Tel. +46 40 333756, Fax +46 40337016

Details: Prof. J.P. Barras, Physiology Institute, University of Basel (Switzerland), Fax+4161267 3559

Details: Dr. Rodney Levick, Secretary British Microcirculation Society, Department of Physiology, St. George's Hospital Medical School, Cranmer Terrace, London SW17 ORE (UK), Tel. 44 (0)81 6729944

Details: Prof. Claudio Allegra, Via S. Erasmo 14,1-00184 Rome (Italy), Tel. +39 6 7705565/7008893, Fax +39 6 70493570/7008893 
Details: Prof. Tellio di Perri, Istituto dí Clinica Medica Generale e Terapia Medica, Università degli Studi di Siena, Nuova Policlinica, Viale Bracci, 1-53100 Siena (Italy), Tel. +39-577 290741, Fax +39-577 44114

Details: Congress Secretariat, Concorde Services, 10 Wendell Road, London|W12 9RT(UK), Tel. +81 743 3106, Fax+81 7431010

Details: Prof. K. Messmer, Institute for Surgical Research, Klinikum Grosshadern, Marchioninistrasse 15, D-81366 Munich (Germany),

Tel. +89 7095 4400, Fax +89 70958897

Organization/exhibition: INCOMA GmbH, Nieder-Röder-Weg 12,

D-63150 Heusenstamm (Germany), Tel. +6104 65024, Fax +6104 67774

Announcement

5-Year Postdoctoral Post in Physiology at St. George’s Hospital Medical School

Department of Physiology, University of London, UK

Postdoctoral research scientist sought under new 5-year Wellcome Programme Grant to study macromolecular-fluid interactions in transport across joint-lining interstitium, a process fundamental to joint function and underpinning arthritis tests. The candidate should have a $\mathrm{PhD}$ in Physiology, Physiological Biochemistry or related subject and a good primary degree. The programme uniquely combines physiological perfusion techniques in vivo (animal knee model) with biochemistry (HPLC) and ultrastructure (electron microscopy), offering acquisition of diverse technical experience. Training is available, supervised by Dr. J.R. Levick (Department of Physiology, St. George's) and Prof. R.M. Mason (Department of Biochemistry, Charing Cross). Career development also includes supervision of postgraduate research and teaching opportunities.

Salary E 20,989 (incl. London Allowance) with annual increments (grade 1A) and annual meetings allowance (E 500). Start negotiable.

Contact:

Personnel (081 784 2791) or Dr. Levick direct (081 6729944 ext. 55354) St. George’s Hospital Medical School Tooting, London SW17 ORE (UK)

195 